\title{
POINT DEFECT SURVIVAL AND CLUSTERING FRACTIONS OBTAINED FROM MOLECULAR DYNAMICS SIMULATIONS OF HIGH ENERGY CASCADES
}

\author{
ROGER E. STOLLER \\ Metals and Ceramics Division \\ Oak Ridge National Laboratory \\ P.O. Box 2008 \\ Oak Ridge, TN 37831-6376 USA
}

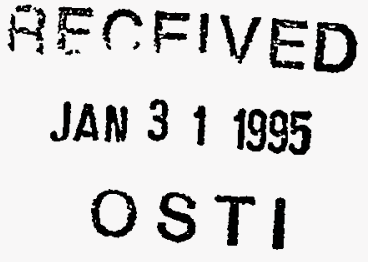

\section{ABSTRACT}

The evolution of high-energy displacement cascades in iron has been investigated for times up to 200 ps using the method of molecular dynamics simulation. The simulations were carried out using the MOLDY code and a modified version of the many-body interatomic potential developed by Finnis and Sinclair. Previously reported results have been supplemented by a series of $10 \mathrm{keV}$ simulations at $900 \mathrm{~K}$ and $20 \mathrm{keV}$ simulations at $100 \mathrm{~K}$. The results indicate that the fraction of the Frenkel pairs escaping in-cascade recombination is somewhat higher and the fraction of the surviving point defects that cluster is lower in iron than in materials such as copper. In particular, vacancy clustering appears to be inhibited in iron. Many of the larger interstitial clusters were observed to exhibit a complex, three-dimensional morphology. The apparent mobility of the $<111>$ crowdion and clusters of $<111>$ crowdions was very high.

\section{INTRODUCTION}

The method of molecular dynamics (MD) has seen broad application to the problem of simulating displacement cascades in irradiated materials [1-7], and the recent evolution of computer technology permits the simulation of higher energy events and higher temperatures with their requirement for larger blocks of atoms. These same computing resources have permitted cascade simulations to be completed in sufficient numbers to allow for statistically meaningful trends to be determined as a function of simulation energy and irradiation temperature, and for comparisons to be made between different materials [5]. For example, the comparison of iron and copper contained in Ref. 5 is based on a database of over 600 cascades. Cascade energies from 60 . $\mathrm{eV}$ to $10 \mathrm{keV}$ were used at irradiation temperatures of 100,600 , and $900 \mathrm{~K}$.

The results presented herein supplement a collaborative study discussed in Ref. 5 . The additional work includes the analysis of seven $10 \mathrm{keV}$ cascades at $900 \mathrm{~K}$ and ten $20 \mathrm{keV}$ cascades at $100 \mathrm{~K}$. In order to investigate cascade evolution, one of the $10 \mathrm{keV}$ cascades was run for $100 \mathrm{ps}$ and one of the $20 \mathrm{keV}$ cascades for $200 \mathrm{ps}$. In addition, one of the $10 \mathrm{keV}, 100 \mathrm{~K}$ cascades from ref. 5 was extended to $100 \mathrm{ps}$. The higher energy provides the opportunity to examine how the trends established at lower energies extrapolate into the domain of significant subcascade 
formation. The motivation for longer simulations is twofold: (1) to verify that the typical cutoff time of 10 to $20 \mathrm{ps}$ is adequate to capture the physical processes at work and (2) to determine the long-term fate of the uncollapsed interstitial clusters that were observed in some of the cascades $[5,6]$.

\section{SIMULATION METHOD}

This work employed the MOLDY computer code and the many-body type interatomic potential originally developed by Finnis and Sinclair for $\alpha$-iron [8] and subsequently modified by Calder and Bacon [3]. The details of this potential and its use are documented elsewhere [1,3,5,8]. The simulations were carried out using an atom block with periodic boundary conditions and at constant pressure. Since the boundary atoms were not damped to remove heat, the initiation of a cascade event led to some lattice heating. The primary impact of the temperature increase (up to $300 \mathrm{~K}$ at $1 \mathrm{ps}$ ) is to extend the lifetime of the thermal spike, it has no impact on the ballistic phase of the cascade. In addition, the temperature increase does not appear to influence defect survival since this has been shown to exhibit very little temperature dependence $[3,5]$.

Prior to conducting the cascade simulations, a block of atoms was thermally equilibrated for about 10 ps to establish the phonon waves. This equilibrated block of atoms was used as the starting point for the subsequent cascade simulations. For the 10 and $20 \mathrm{keV}$ simulations discussed here, the atom blocks contained 250,000 atoms. The simulations were initiated by giving one of the lattice atoms the defined amount of kinetic energy in a specific direction. Multiple cascades were differentiated by changing the location of the initial primary knock-on atom (PKA). As discussed in Ref. 5, this initial kinetic energy is analogous to the damage energy in the standard NRT displacement model [9]. In the terminology of the NRT model, a $10 \mathrm{keV}$ cascade simulation energy (or damage energy) corresponds to a PKA energy of $13.7 \mathrm{keV}$ [5,9]. The difference between these two values is the kinetic energy lost to electronic excitation; this energy loss mechanism is not simulated in the MOLDY code [5].

\section{SIMULATION RESULTS}

The number of surviving point defects, $N_{F P}$, has been shown to exhibit a simple power-law dependence on the $\mathrm{MD}$ simulation energy, $E_{M D}$ in $\mathrm{keV}$, for a number of materials [7]. In the case of iron cascades up to $10 \mathrm{keV}$ at a temperature of $100 \mathrm{~K}$ :

$$
N_{F P}=5.67 \cdot E_{M D}^{0.779}
$$

Ten $20 \mathrm{keV}$ cascade simulations were run at $100 \mathrm{~K}$ to test whether or not this relationship would hold as the cascade energy increased into the domain of significant subcascade formation. These simulations were typically run for $15 \mathrm{ps}$ and then quenched to $\sim 0 \mathrm{~K}$ prior to characterizing the number and configuration of the residual defects. Although little change was observed in the 
defect structure after about $10 \mathrm{ps,} \mathrm{one} \mathrm{simulation} \mathrm{was} \mathrm{extended} \mathrm{to} 200 \mathrm{ps}$ to examine the cascade evolution over this longer time.

An example of the subcascade formation observed in the $20 \mathrm{keV}$ cascades is shown in Fig. 1 . Several subcascade lobes can be observed that formed due to either high-angle scattering events or short-range channeling. On average, the degree of subcascade formation at $20 \mathrm{keV}$ is much greater than observed at $10 \mathrm{keV}$; essentially no significant subcascade formation is observed at energies below about $10 \mathrm{keV}$. In spite of the differences in cascade morphology, the average number of surviving defects at $20 \mathrm{keV}$ and $100 \mathrm{~K}$ falls precisely on an extrapolation of Eq. 1 which was fit to the results obtained between 0.5 and $10 \mathrm{keV}$. This is shown in Fig. 2a, where the present results are plotted along with the lower energy data. The effect of temperature on defect formation appears to be modest in Fig. 2a. The values for 600 and $900 \mathrm{~K}$ seem to follow the same parallel line, slightly below the $100 \mathrm{~K}$ line.

The data for energies below $0.5 \mathrm{keV}$ fall above the line in Fig. $2 \mathrm{a}$ because a well developed collision cascade does not form at these energies [5]. The data from Fig. $2 a$ are normalized to the NRT [9] number of displacements and replotted in Fig. 2b. Even at the highest energies, about $25 \%$ of the NRT value survive in-cascade recombination. This is somewhat more than observed in copper [5]. As shown in Fig. 3, the fraction of the surviving interstitials that are found in clusters containing two or more interstitials can also be smoothly extrapolated from the lower energy results. The lines in Fig. 3 are only meant to be a guide for the eye. The clustering fraction exhibits much more scatter than the number of surviving defects, particularly at lower energies. The notable absence of in-cascade vacancy cluster formation discussed in Ref. 5 also persists for the $10 \mathrm{keV}$ cascades at $900 \mathrm{~K}$ and the $20 \mathrm{keV}$ cascades at $100 \mathrm{~K}$. The clustering fraction at $900 \mathrm{~K}$ increased sharply above $5 \mathrm{keV}$ to $70 \%$ for $10 \mathrm{keV}$.

Observation of the 10 and $20 \mathrm{keV}$ cascades that were continued for long times revealed that some rather large interstitial clusters exhibited unexpectedly high mobility. In one example at 20 $\mathrm{keV}$, a loose cluster of eight interstitials was observed to thermally reorient itself for a few ps until all the interstitials were $<111>$ crowdions. Once this was achieved, this cluster of crowdions exhibited very high mobility, gliding over a long distance until it encountered another interstitial and several crowdions were defocused. Similar examples of highly mobile interstitial ciusters were observed in several 10 and $20 \mathrm{keV}$ cascades. No attempt has yet been made to determine the activation energy for migration of such clusters, but their mobility at a lattice temperature of less than $150 \mathrm{~K}$ indicates that it is probably only a few tenths of an $\mathrm{eV}$.

One unusual observation reported in Ref. 5 was that some of the interstitial clusters exhibited a 3-dimensional morphology rather than collapsing into planar dislocation loops as seen in materials such as copper [2]. One possible explanation was that 10 to $15 \mathrm{ps}$ was not sufficient time for the cluster to reorient and collapse [3]. To examine this possibility, one of the $100 \mathrm{~K}, 10 \mathrm{keV}$ cascades that contained such a cluster of eight interstitials was extended to $100 \mathrm{ps}$. Very little restructuring was seen over the time from 10 to $100 \mathrm{ps.} \mathrm{In} \mathrm{fact,} \mathrm{the} \mathrm{cluster} \mathrm{had} \mathrm{coalesced} \mathrm{into}$ 
nearly its final configuration by $10 \mathrm{ps}$. This particular cluster of eight interstitials was examined in detail by searching a large number of low order crystallographic projections in an attempt to find a projection in which it would appear as a loop. Such a projection could not be found. Rather, the cluster was clearly three-dimensional with a di-, tri-, and another di-interstitial on adjacent, closepacked (110) planes as shown in Fig. 4. The eighth interstitial is a [110] dumbbell that lies perpendicular to the others in Fig. 4a. Figs. 4(b-d) are [101] projections through the three center (101) planes in Fig. 4a. Since the interstitial geometry in $\alpha$-iron is either a dumbbell or crowdion, the criteria used to detect point defects in the MD code [5] leads to a single interstitial being displayed as a defect complex containing what appears to be two interstitials separated by a vacancy. The actual number of interstitials is obtained by subtracting the apparent vacancies from the number of apparent interstitials.

\section{DISCUSSION}

The results obtained from the $20 \mathrm{keV}$ cascade simulations could be smoothly extrapolated from the lower energy simulations reported previously. The surprisingly good power-law fit to the number of surviving defects from 0.5 to $20 \mathrm{keV}$ was unexpected since subcascade formation could have caused some deviation. No simple physical rationale for this power-law dependence exists. The simple damage energy concept embodied in the NRT displacement model [9] leads to an exponent of 1.0. With the determined exponent of 0.779 , the ratio of the MD surviving displacements to the NRT displacements decreases as the damage energy increases. However, this fraction remains above 0.25 out to the highest energies. The number of surviving defects is relatively insensitive to temperature [5].

A $20 \mathrm{keV}$ damage energy in iron corresponds to a $29 \mathrm{keV}$ PKA energy, which is the average PKA energy for scattering by a $0.83 \mathrm{MeV}$ neutron. Since only about 12 to $20 \%$ of the neutrons in the core of a typical light water or sodium cooled reactor have energies above $0.83 \mathrm{MeV}$, most of the energy domain of interest to fission reactor materials has been explored by the $60 \mathrm{eV}$ to 20 $\mathrm{keV}$ cascade simulations presented here and in Ref. 5 . In addition, the smooth energy dependence shown in Figs. 2 and 3 suggests that these data will be representative of the higher energy neutrons that make up part of a deuterium-tritium fusion reactor spectrum.

Finally, the data presented in this paper can be used to speculate about two interesting possibilities. The first has to do with the three-dimensional character of the interstitial clusters that were observed, and the second is related to the apparent mobility of the interstitial clusters. Of course, this speculation is only reasonable to the extent that the interatomic potential is reproducing the "real" behavior of the material. For example, it is not clear how much the absence of electron-phonon coupling influences the results of these MD simulations [5].

It is well known that ferritic steels exhibit a great deal of hardening at very low doses, well before any radiation-induced defects are visible by transmission electron microscopy. In this case, the features responsible for the hardening must be smaller than about $1 \mathrm{~nm}$. A point defect cluster 
of the type shown in Fig. 4 is a plausible candidate for the invisible hardening center. Since the interstitials are distributed over several adjacent close-packed planes, the maximum linear dimension of the cluster is smaller than if the same number of interstitials were contained in a two-dimensional defect such as a faulted loop. At the same time, the strain field associated with this cluster may make it a very effective hardening agent. Thus, these interstitial clusters that are formed directly by the cascade event could lead to significant hardening before growing to a visible size as dose is accumulated.

The degree to which interstitial cluster mobility was observed to vary with size and orientation could help explain some of the structure in defect annealing curves. It may be that the interpretive differences that originally led to the well-known controversy over the one- and twointerstitial models [10] arose in part as a result of the confounding influence of such small, mobile interstitial clusters. Perhaps the analysis should not have been focused on the mobility of monointerstitials alone, but rather on a broader range of defects. The high mobility exhibited by the multiple crowdion should remove point defects very efficiently at low temperatures, leading to a low apparent migration energy. With multiple cluster sizes, a fairly broad or structured annealing peak should be observed. The impact of these mobile interstitial clusters is currently being investigated using a kinetic model of ferritic steel embrittlement.

\section{ACKNOWLEGEMENTS}

The results of the earlier collaboration with Drs. W. J. Phythian and A. J. E. Foreman of the Harwell Laboratory and Drs. D. J. Bacon and A. F. Calder of the University of Liverpool, and helpful discussions with Dr. S. J. Zinkle of ORNL are gratefully acknowledged. This research is sponsored by the Office of Nuclear Regulatory Research, U.S. Nuclear Regulatory Commission under interagency agreement DOE 1886-8109-8L with the U.S. Department of Energy and by the Division of Materials Sciences, U.S. Department of Energy under contract DE-AC05$840 R 21400$ with Martin Marietta Energy Systems, Inc. The purchase of a workstation used to conduct many of these calculations was supported by the Office of Fusion Energy, U.S. Department of Energy.

\section{REFERENCES}

1. R. S. Averback, T. Diaz de la Rubia, and R. Benedek, Nucl. Inst. and Meth. B33, 693 (1988).

2. A. J. E. Foreman, W. J. Phythian, and C. A. English, Phil. Mag. A66, 571 (1992).

3. A. F. Calder and D. J. Bacon, J. Nucl. Mater 207, 25 (1993).

4. D. J. Bacon and T. Diaz de la Rubia, J. Nucl. Mater 216, 275 (1994).

5. W. J. Phythian, R. E. Stoller, A. J. E. Foreman, A. F. Calder, and D. J. Bacon, J. Nucl. Mater. 223 (1995) 245-261.

6. R. E. Stoller, "Molecular Dynamics Simulations Of High Energy Cascades", in Microstructure of Irradiated Materials, eds. I. M. Robertson, L. E. Rehn, S. J. Zinkle, and 
W. J. Phythian (Materiais Research Society, Pittsburgh, 1995) pp. 21-26.

7. D. J. Bacon, A. F. Calder, F. Gao, V. G. Kapinos, and S. J. Wooding, Nucl. Inst. and Meth. B102 (1995) 37-46.

8. M. W. Finnis and J. E. Sinclair, Phil. Mag. A50, 45 (1984).

9. M. J. Norgett, M. T. Robinson, and I. M. Torrens, Nucl. Eng. and Des. 33, 50 (1975).

10. F. W. Young, Jr., J. Nucl. Mater. 69 \& 70, 310 (1978). 


\section{Figure Captions}

Fig. 1 Example of subcascade formation in a $20 \mathrm{keV}$ cascade simulation at $100 \mathrm{~K}$.

Fig. 2 Energy dependence of the number of surviving point defects (a) and the fractional survival, relative to the NRT model (b).

Fig. 3 Energy dependence of the fraction of surviving interstitials that are in clusters.

Fig. 4 Three-dimension interstitial cluster in $10 \mathrm{keV}$ cascade, (a) [010] projection normal to five adjacent $\{110\}$ planes and $(b-d)$ projections through three of the $\{110\}$ planes. 


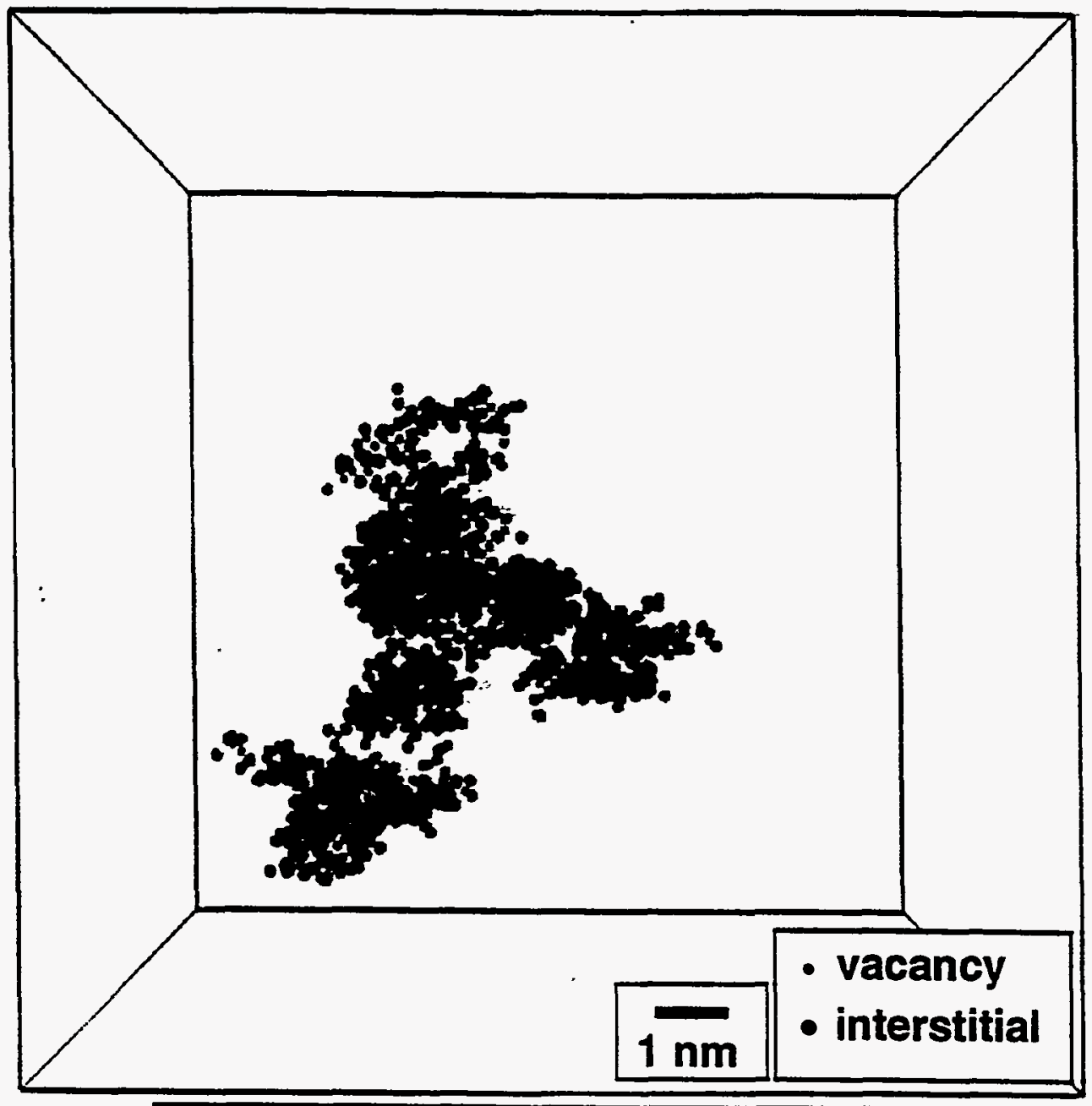

$20 \mathrm{keV}$ cascade $\mathrm{Fe}, 100 \mathrm{~K}, \mathrm{t}=0.1 \mathrm{ps}$ 


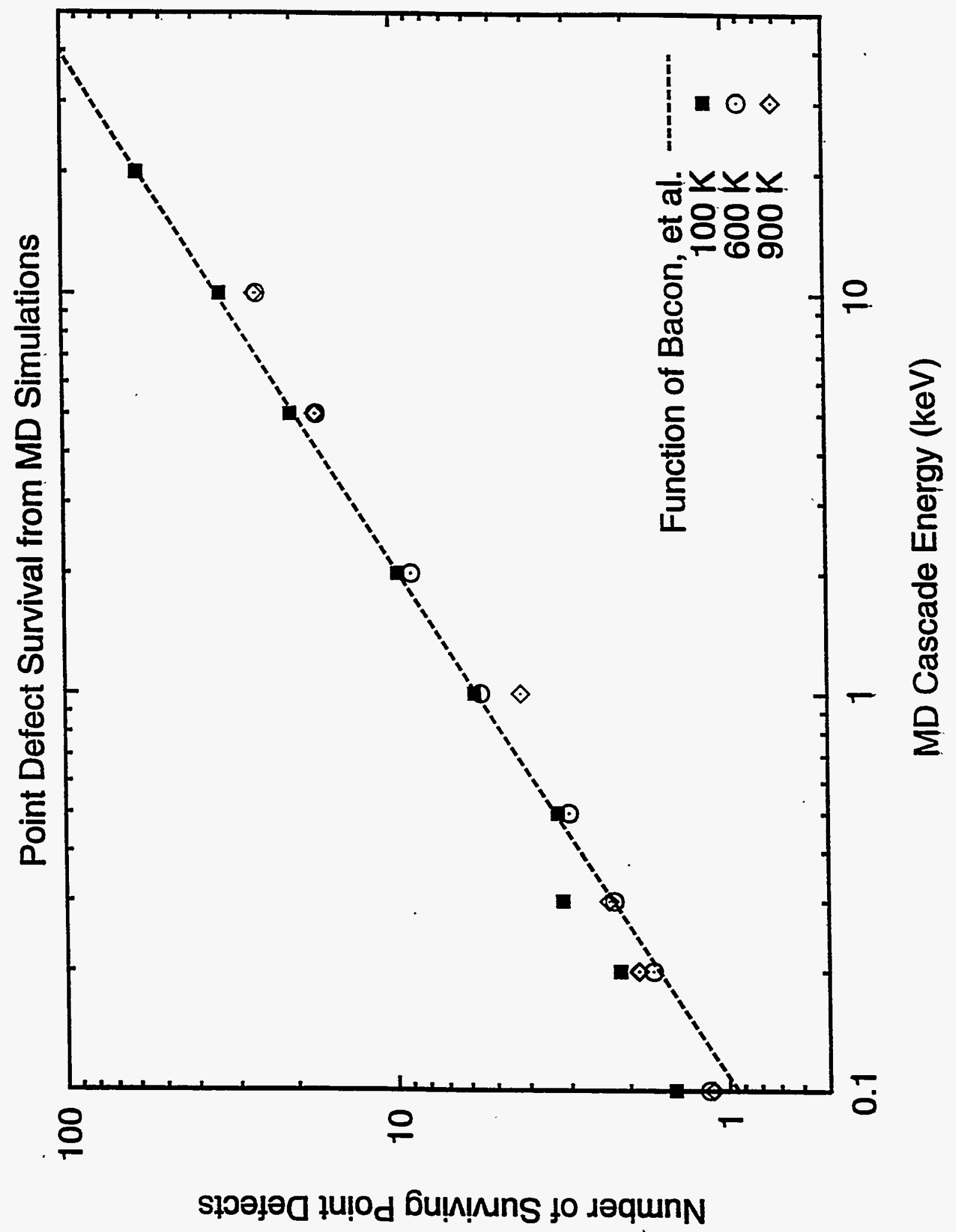




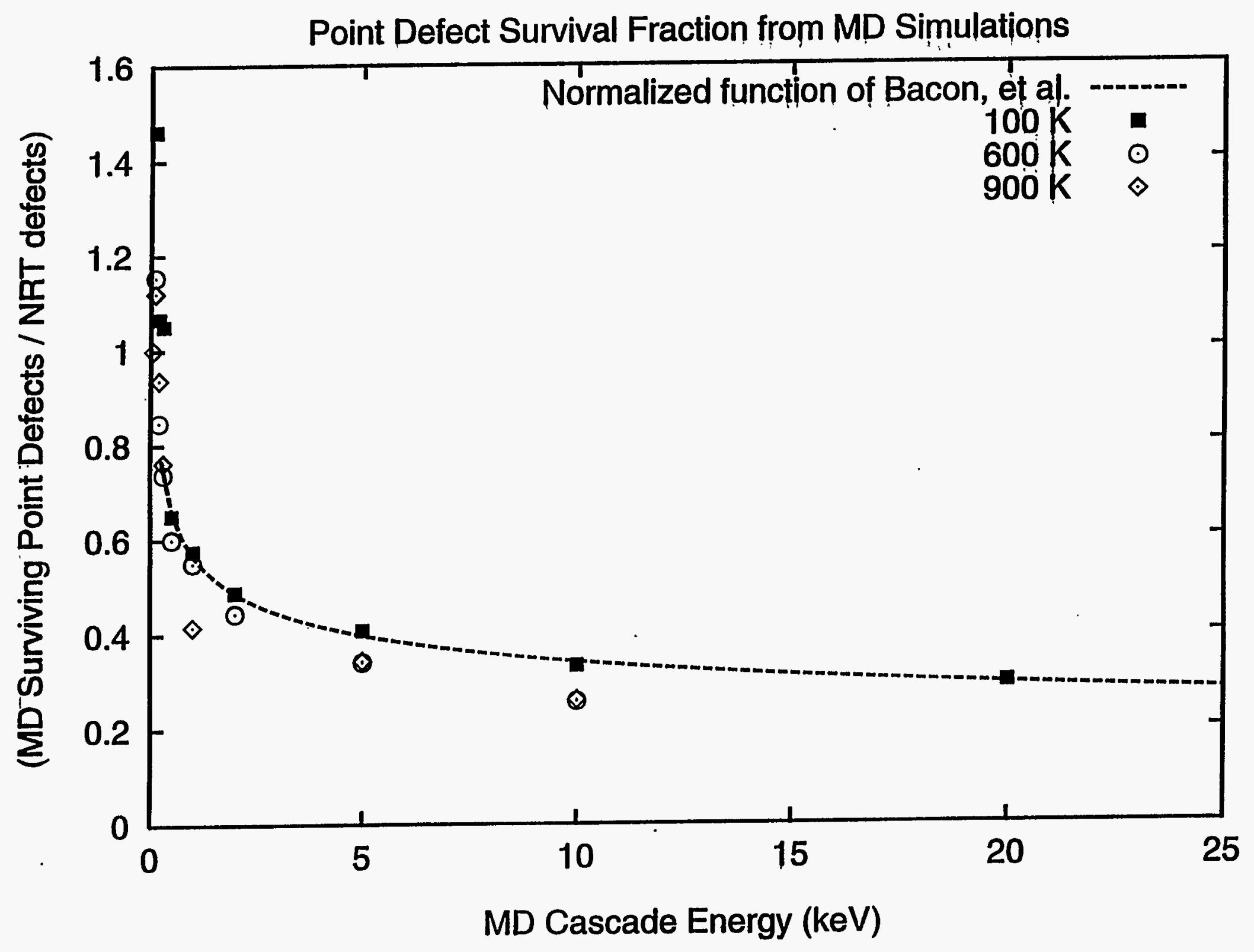

Stoller, fig 26 


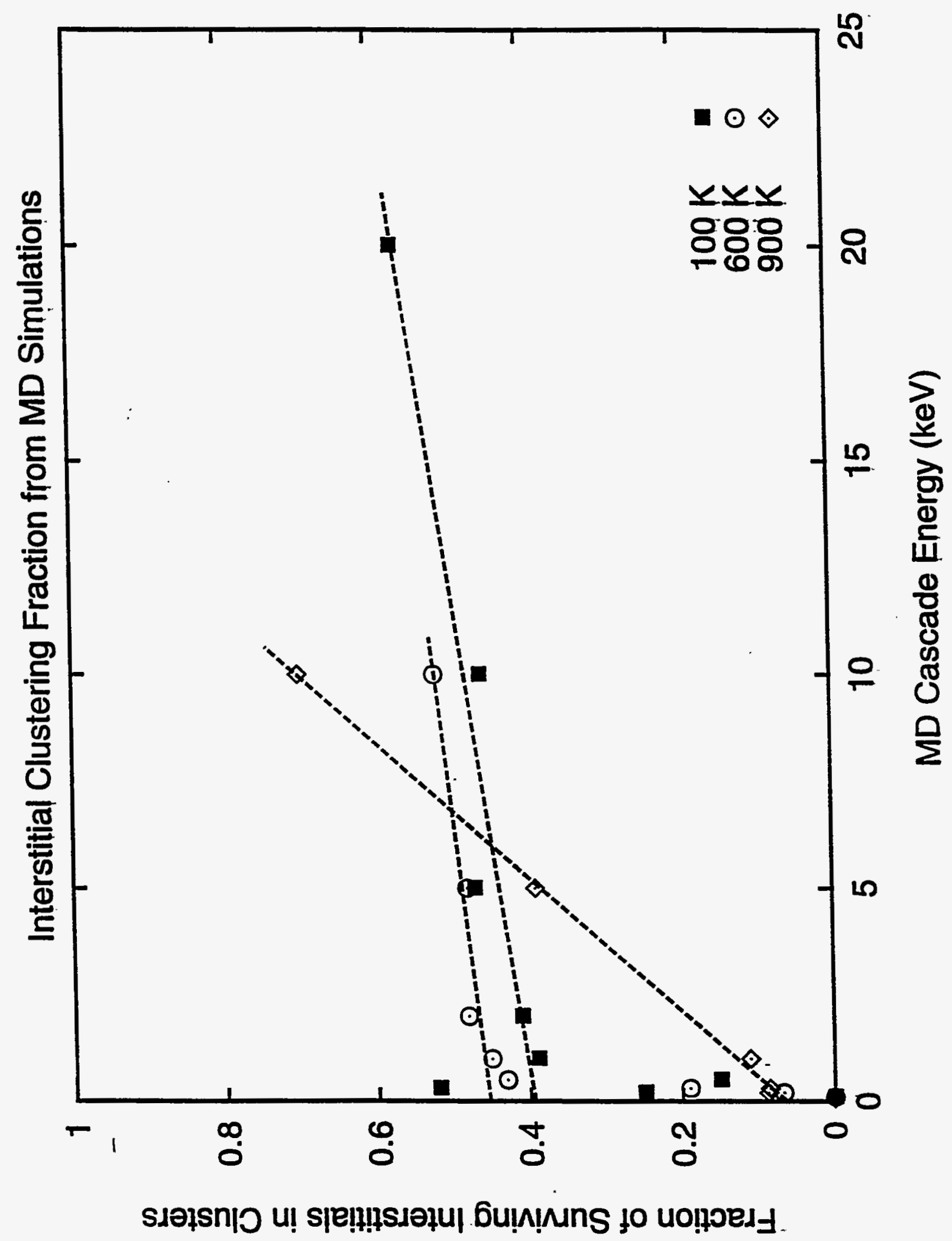


Structure of 8-interstitial cluster from $10 \mathrm{keV}$ cascade in iron at $100 \mathrm{~K}$

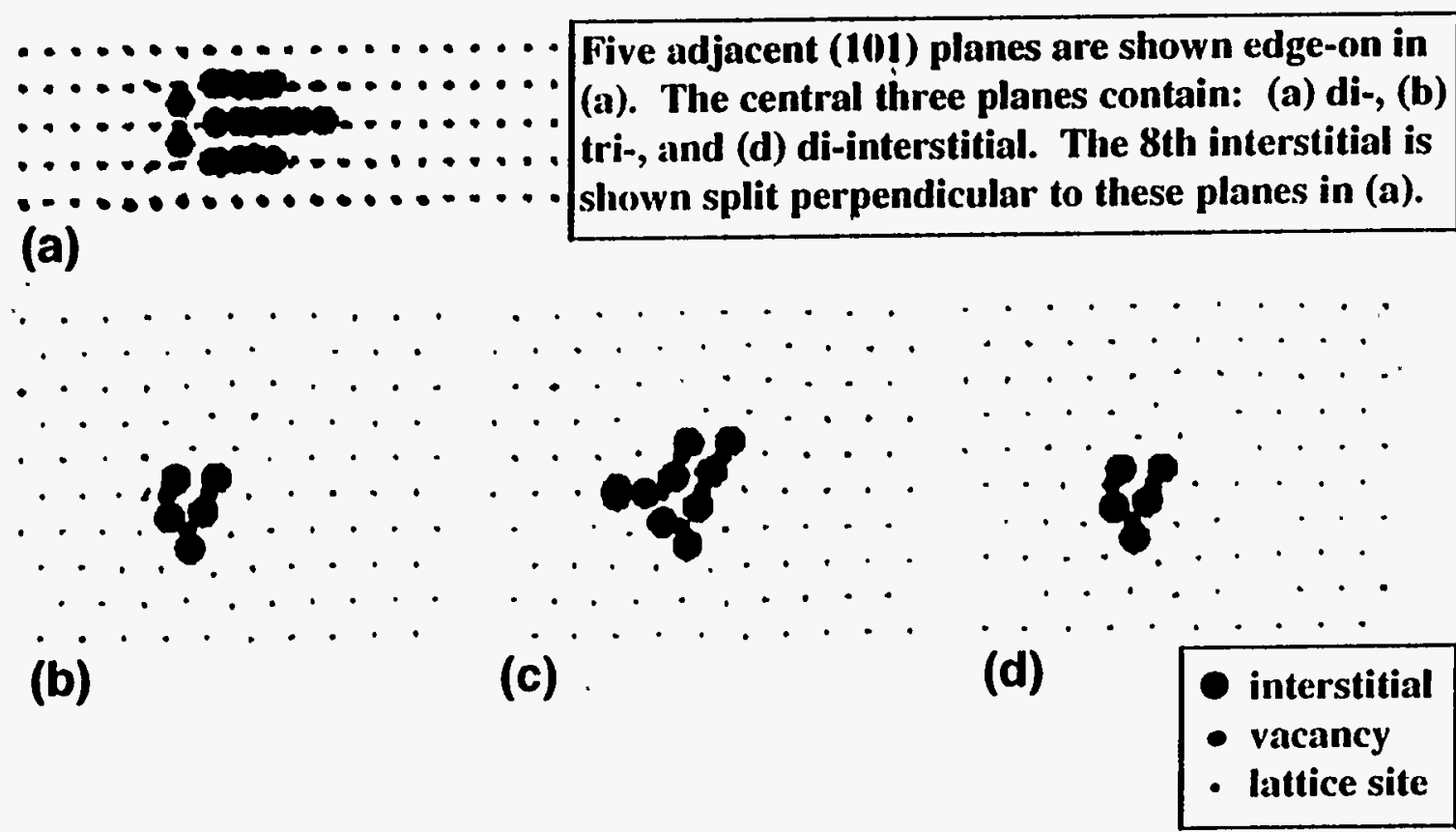

\section{DISCLAIMER}

This report was prepared as an account of work sponsored by an agency of the United States Government. Neither the United States Government nor any agency thereof, nor any of their employees, makes any warranty, express or implied, or assumes any legal liability or of their bility for the accuracy, completeness, or usefulness or assumes any legal liability or responsiprocess disclosed, or represents that its use would not infringe privately owned rights. Reference herein to any specific commercial product, process, or service by trade name, trademark manufacturer, or otherwise does not necessarily constitute or imply its endorsementemark, mendation, or favoring by the United States Govertitute or imply its endorsement, recommendation, or favoring by the United States Government or any agency thereof. The views and opinions of authors expressed herein do not necessarily state or reflect those of the United States Government or any agency thereof. 